\title{
Spin-Dependent On-Site Electron Correlations and Localization in Itinerant Ferromagnets
}

\author{
R. Gotter, ${ }^{1}$ G. Fratesi, ${ }^{2}$ R. A. Bartynski, ${ }^{3}$ F. Da Pieve, ${ }^{4}$ F. Offi, ${ }^{5}$ A. Ruocco, ${ }^{5}$ S. Ugenti, ${ }^{5}$ M. I. Trioni, ${ }^{6}$ \\ G.P. Brivio, ${ }^{2}$ and G. Stefani ${ }^{5}$ \\ ${ }^{1}$ CNR, Istituto Officina dei Materiali (IOM), Area Science Park, I-34149 Basovizza-Trieste, Italy \\ ${ }^{2}$ ETSF, CNISM, and Dipartimento Scienza dei Materiali, Università Milano Bicocca, I-20125 Milano, Italy \\ ${ }^{3}$ Department of Physics and Astronomy and Laboratory of Surface Modification, Rutgers University, \\ Piscataway, New Jersey 08854, USA \\ ${ }^{4}$ EMAT, University of Antwerp, B-2020 Antwerp, Belgium \\ ${ }^{5}$ CNISM and Dipartimento di Fisica, Università Roma Tre, I-00146 Rome, Italy \\ ${ }^{6}$ CNR-National Research Council of Italy, ISTM, I-20133 Milano, Italy
}

(Received 12 July 2012; published 17 September 2012)

\begin{abstract}
Spin selectivity in angle-resolved Auger photoelectron coincidence spectroscopy (AR-APECS) is used to probe electron correlation in ferromagnetic thin films. In particular, exploiting the AR-APECS capability to discriminate Auger electron emission events characterized by valence hole pairs created either in the high or in the low total spin state, a strong correlation effect in the $\mathrm{Fe} M_{2,3} V V$ Auger line shape (measured in coincidence with the $\mathrm{Fe} 3 p$ photoelectrons) of $\mathrm{Fe} / \mathrm{Cu}(001)$ thin films is detected and ascribed to interactions within the majority spin subband. Such an assignment follows from a close comparison of the experimental AR-APECS line shapes with the predictions of a model based on spin polarized density functional theory and the Cini-Sawatzky approach.
\end{abstract}

PACS numbers: 71.10. $-\mathrm{w}, 75.70 .-\mathrm{i}, 79.60 .-\mathrm{i}, 82.80 . \mathrm{Pv}$

The nature of valence electrons in solids is often classified in terms of the degree to which electron-electron interactions dictate their behavior. Itinerant electron systems, particularly those whose wave functions are derived from states of $s$ - or $p$-character, are often characterized by an on-site electron-electron Coulomb interaction energy $(U)$ that is small compared to the valence band width $(W)$, and are well described within a mean field approximation using approaches such as density functional theory [1]. On the other hand, for systems where $U$ is large compared to $W$, neglect of the mutual repulsion among the electrons will lead to an erroneous description of the material's properties. Examples of such highly correlated systems are those where $f$-electrons dictate material properties: Electron-electron interactions are so strong that they are best described by approaches that embrace these correlations at the outset, such as dynamical mean field theory [2,3]. Transition metals and their compounds often are intermediate cases where electron-electron interactions cannot be ignored, but may be adequately described in a perturbative manner [4-7]. When the Coulomb repulsion among electrons is important, it is typically characterized by a single average interaction energy $U$. However, in ferromagnetic systems where the magnetic exchange interaction energy, $\Delta$, is of a similar magnitude as $U$ and $W$, majority and minority spin electrons may exhibit different degrees of localization [8,9]. As a result, different theoretical approaches must be used to adequately describe the behavior of each spin population [10].

In this Letter, we investigate this issue with a combined experimental and theoretical angle-resolved
Auger-photoelectron coincidence spectroscopy (ARAPECS) study of the $\mathrm{Fe} M_{2,3} V V$ Auger transition from a ferromagnetic $\mathrm{Fe}$ thin film epitaxially grown on the $\mathrm{Cu}$ (001) surface. The final state in this core-valence-valence Auger transition is comprised of two holes in the $\mathrm{Fe}$ valence band. By appropriate choice of experimental parameters, sensitivity of the AR-APECS spectra to triplet (total spin moment $S=1$ ) configurations of the two-hole final state can be enhanced or reduced with respect to that of the singlet $(S=0)$ [11]. We find that, while contributions to the spectrum from decay channels that involve minority spin electrons are well described within an independent electron approximation, significant correlation effects must be included to account for the final state with two majority-spin holes.

The experiments were carried out at the ALOISA beam line of the ELETTRA synchrotron radiation facility (Basovizza-Trieste, Italy). For the measurements, performed at room temperature, we used a $\mathrm{Cu}(001)$ substrate, cleaned in the vacuum chamber by $1 \mathrm{keV} \mathrm{Ar}^{+}$-ion bombardment and subsequently annealed to $\sim 700 \mathrm{~K}$. An atomically clean and highly ordered $\mathrm{Cu}(001)$ surface was verified by $\mathrm{x}$-ray photoemission spectroscopy and reflection high energy electron diffraction. A three monolayer thick Fe film was grown by electron beam-assisted thermal evaporation at a pressure of about $5 \times 10^{-8} \mathrm{~Pa}$. The film thickness was monitored by calibrating the evaporation rate with a quartz microbalance, and the film was not subjected to any magnetic or thermal treatment. Monochromatic, linearly polarized photons of $250 \mathrm{eV}$ energy impinged on the sample at grazing incidence of 
about $5^{\circ}$, with the sample normal in the plane defined by $\vec{\epsilon}$, the light polarization, and $\vec{k}$, its propagation vector. In the experimental set up, discussed in detail in a previous paper [12], five electron energy analyzers, each having an angular resolution of about $2^{\circ}$, were positioned in the plane defined by $\vec{\epsilon}$ and $\vec{k}$; one electron analyzer was aligned with $\vec{\epsilon}$, two analyzers were at $\pm 18^{\circ}$ with respect to $\vec{\epsilon}$ and two at $\pm 36^{\circ}$. For the present experiment the two analyzers at $\pm 18^{\circ}$ were tuned to detect $\mathrm{Fe} 3 p$ core level photoelectrons within an energy window of $4.3 \mathrm{eV}$, fixed by the analyzer energy resolution, suitable for collecting the entire $\mathrm{Fe} 3 p$ peak. The other analyzers were scanning over the $M_{2,3} V V$ Fe Auger electrons spectra with an energy resolution of $1.8 \mathrm{eV}$. Each pair of analyzers can be considered arranged according to one of two geometries: the photoelectron emission direction was always set close (at $18^{\circ}$ ) from the light polarization direction (which is taken as the quantization axis of the angular momentum $l$ ), hence favoring the detection of photoelectrons having $m_{l}=0$, henceforth termed "aligned" [13]; the Auger electron emission was either aligned (at $0^{\circ}$ ) or "not aligned" (i.e., at a larger angle, $36^{\circ}$ ) with the light polarization. The two configurations will be referred to as $A A$, meaning that both electrons are aligned (A) with $\vec{\epsilon}$ and $A N$, where the photoelectron is aligned $(A)$ with $\vec{\epsilon}$, and the Auger electron is not $(N)$. The azimuthal directions were along the (100) symmetry axis of the surface.

The Fe $M_{2,3} V V$ Auger spectra taken at room temperature (which is below the critical temperature for the used Fe thickness [14]) in coincidence with $3 p$ photoelectrons are reported in Fig. 1 for the $A A$ (open circles) and $A N$ (solid triangles) geometries. The solid lines are guides to the eye. The single (i.e., noncoincidence) Auger spectra, taken simultaneously in the two geometries, are indistinguishable from each other and their sum is shown as

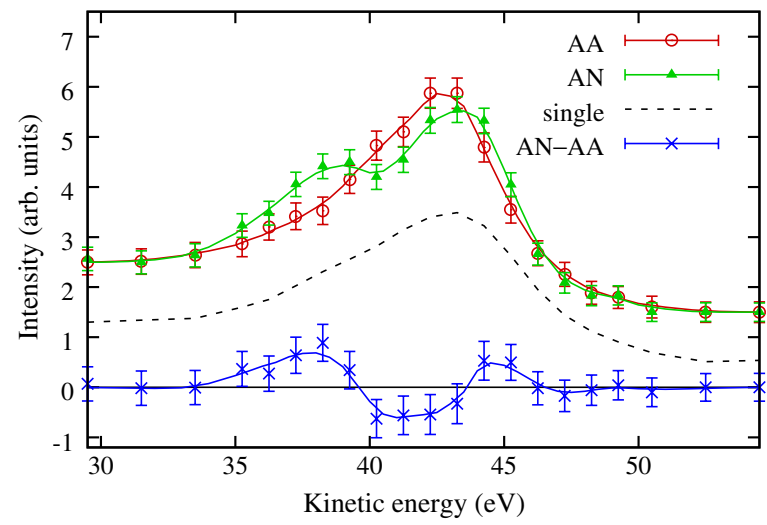

FIG. 1 (color online). $\quad$ Fe $M_{2,3} V V$ Auger spectra from a trilayer of $\mathrm{Fe} / \mathrm{Cu}(001)$ film taken at room temperature in coincidence with the Fe $3 p$ photoelectrons (symbols with error bars and solid lines) and simultaneously acquired single spectra (dashed lines). Open circles (solid triangles) refer to $A A(A N)$ geometry. The lower curve shows the $A N-A A$ difference. the dashed line. The line shape of the $A N$ coincidence spectrum clearly exhibits two features centered at about 38 and $43 \mathrm{eV}$. These features are reduced and enhanced, respectively, in the $A A$ spectrum. The subtraction between the two spectra, reported as crosses at the bottom of Fig. 1, highlights the differences incurred by changing geometry. In previous AR-APECS studies of $\mathrm{Sn}$ [11] and $\mathrm{Cu}$ [13] it was found that changing from the $A A$ to the $A N$ geometry enhances the high spin (triplet) double hole Auger final states and reduces the low spin (singlet) states. Based on those results, we associate the $38 \mathrm{eV}$ and $43 \mathrm{eV}$ features with triplet and singlet final states, respectively. This qualitative interpretation will be placed on a solid quantitative footing here by studying theoretically the angular resolved transition as a function of the spin configuration.

We assume that the initial photoionization and the subsequent Auger decay of the core hole can be treated as two independent events, the latter being described by the Fermi golden rule. We evaluate the density of states (DOS) and wave functions of the final state in absence of the core hole [15] and compute the total spectrum for electrons emitted with momentum $\mathbf{k}_{A}$ and kinetic energy $E_{k_{A}}$ as

$$
P\left(\mathbf{k}_{A}\right)=2 \pi \sum_{X Y} A_{X}^{*} A_{Y} D_{X Y}\left(E_{C}+E_{k_{A}}\right),
$$

where $X$ and $Y$ are the final-state quantum numbers, $A_{X}$ is the Auger matrix element (given by the Coulomb operator $\hat{V})$ corresponding to the final state $X, E_{C}$ is the core electron energy level here taken empirically as $E_{C}=$ $-46.0 \mathrm{eV}, D_{X Y}$ represents the two-electron DOS, and atomic units are used unless otherwise specified. In the present treatment we neglect the relatively small spin-orbit interactions both in the core $3 p$ and in the valence $3 d$ states involved in the $M_{2,3} V V$ transition.

Let us expand $X$ by quantum numbers $I, \sigma_{I}, J, \sigma_{J}$, and $Y$ by $I^{\prime}, \sigma_{I^{\prime}}, J^{\prime}, \sigma_{J^{\prime}}$, where $I, J$ denote collectively the singleparticle quantum numbers but for the spins $\sigma_{I}, \sigma_{J}$. The DOS for two noninteracting holes is obtained from the convolution (indicated by $*$ ) of single-particle ones, $d_{I I^{\prime}}^{\left(\sigma_{I}\right)} \delta_{\sigma_{I} \sigma_{I^{\prime}}}$, which are taken to be spin-diagonal:

$$
\begin{aligned}
D_{X Y}= & d_{I I^{\prime}}^{\left(\sigma_{I}\right)} * d_{J J^{\prime}}^{\left(\sigma_{J}\right)} \delta_{\sigma_{I} \sigma_{I^{\prime}}} \delta_{\sigma_{J} \sigma_{J^{\prime}}} \\
& -d_{J I^{\prime}}^{\left(\sigma_{J}\right)} * d_{I J^{\prime}}^{\left(\sigma_{I}\right)} \delta_{\sigma_{J} \sigma_{I^{\prime}}} \delta_{\sigma_{I} \sigma_{J^{\prime}}}
\end{aligned}
$$

Equation (1) may be rewritten by translating the sum over $X$ into $\frac{1}{2} \sum_{I \sigma_{I} J \sigma_{J}}$, and following some steps of calculations we obtain

$$
\begin{aligned}
P_{\sigma_{C} \sigma_{A}}\left(\mathbf{k}_{A}\right)= & 2 \pi \sum_{I J I^{\prime} J^{\prime}}\left[(C A|\hat{V}| I J)-(C A|\hat{V}| J I) \delta_{\sigma_{C} \sigma_{A}}\right] \\
& \times d_{I I^{\prime}}^{\left(\sigma_{C}\right)} * d_{J J^{\prime}}^{\left(\sigma_{A}\right)}\left(E_{C}+E_{k_{A}}\right)\left(I^{\prime} J^{\prime}|\hat{V}| C A\right),
\end{aligned}
$$

where $\mid I J)=|I\rangle \otimes|J\rangle$ are nonantisymmetrized states of the canonical basis [16]; $C$ and $A$ denote quantum numbers of the core electron and of the Auger electron, respectively. 
Recalling that the photoelectrons in the experiments were detected close to the electric field direction $\vec{\epsilon}$, they are assumed to be in a $m_{l}=0$ state. The dipole selection rules of photoemission imply that the core state is also in a $m_{l}=0$ state. Hence, a $3 p_{z}$ core state (with $z$ set along $\overrightarrow{\boldsymbol{\epsilon}}$ ) is taken when evaluating the angular distribution of Auger electrons. To better highlight the angular dependence of the Auger deexcitation, we initially treat the system as a magnetic $\mathrm{Fe}$ atom hosted in infinite $\mathrm{Cu}$ metal modeled by jellium [17]. The Auger intensity $I_{\sigma_{C} \sigma_{A}}$ is obtained by integrating $P_{\sigma_{C} \sigma_{A}}$ in Eq. (3) over the full kinetic energy range and only depends on the polar angle $\theta$ between $\mathbf{k}_{A}$ and the $z$ axis. The results are reported in Fig. 2(a) for the processes resulting from the four possible core- $(C)$ and Auger- $(A)$ electron spin combinations, which we denote by $\left(\sigma_{C} \sigma_{A}\right)$. The curves of Fig. 2(a) exhibit several interesting features. Essential for the present analysis is that $I(\theta)$ for antiparallel spin transitions decreases with $\theta$, while the parallel spins exhibit the opposite angular dependence. This result is the origin of the so-called dichroic effect found in the angular distribution [11,13], represented in Fig. 1 as the difference curve.

To simulate the AR-APECS spectrum, we extract the angular dependence of the matrix elements from the full spectrum calculation assuming that the core-valencevalence signal is proportional to the local two-particle DOS computed in a volume $V$ around the ionized atom, which reflects the localized nature of the process:

$$
P_{\sigma_{C} \sigma_{A}}\left(\mathbf{k}_{A}\right) \approx d_{V}^{\left(\sigma_{C}\right)} * d_{V}^{\left(\sigma_{A}\right)}\left(E_{C}+E_{k_{A}}\right)\left|\mathcal{M}_{\sigma_{C} \sigma_{A}}(\theta)\right|^{2} .
$$

This defines implicitly an "effective matrix element", $\mathcal{M}$, that can be straightforwardly computed from the Auger intensity shown in Fig. 2(a). Indeed, by integrating Eq. (4) over $E_{k_{A}}$ we obtain:

$$
\left|\mathcal{M}_{\sigma_{C} \sigma_{A}}(\theta)\right|^{2}=\frac{I_{\sigma_{C} \sigma_{A}}(\theta)}{n_{V}^{\left(\sigma_{C}\right)} n_{V}^{\left(\sigma_{A}\right)}} .
$$

Here, $n_{V}^{(\sigma)}$ is the valence electron charge of spin $\sigma$ in the atomic volume $V$ (taken to be a sphere of radius $1.77 \AA$ ).
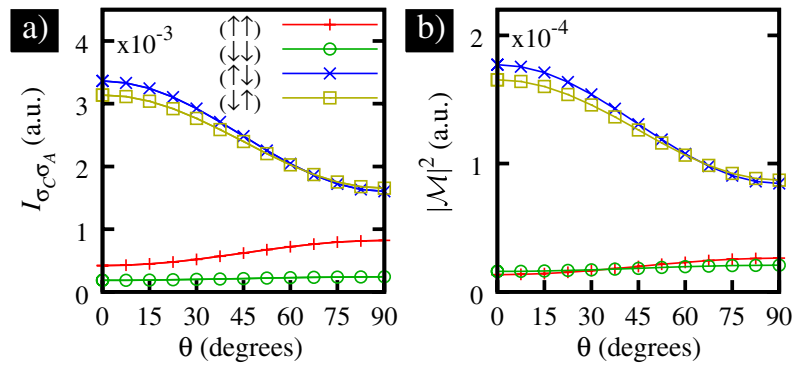

FIG. 2 (color online). $\quad M_{2,3} V V$ Auger intensity, panel (a), and effective squared matrix element as defined in Eq. (5), panel (b), assuming a $3 p_{z}$ core state. Results are shown for different combinations of the electron spins involved, $\left(\sigma_{C} \sigma_{A}\right)$, as indicated in the legend.
The result is reported in Fig. 2(b). Notice that $\mathcal{M}_{\uparrow}$ and $\mathcal{M}_{\Downarrow}$ are very similar, the difference between the respective intensities shown in Fig. 2(a) being attributed to the lower number of the minority spin electrons, $n_{V}^{\downarrow}=3.4$ compared to $n_{V}^{\uparrow}=5.6$.

We are now in the position to evaluate the Auger line shape of the real system by using Eq. (4) with values of $\mathcal{M}$ extracted above. The electronic structure of an Fe trilayer in a planar geometry on a semi-infinite $\mathrm{Cu}$ substrate was calculated with the linearized augmented plane wave embedding approach within density-functional theory [18]. The DOS for the topmost Fe atom is shown in the inset of Fig. 3(a), separately for the majority $(\uparrow)$ and minority $(\downarrow)$ spin components. Note that the DOS for second- and thirdlayer $\mathrm{Fe}$ atoms, which also contribute to the Auger rate, are nearly identical to that from the first layer and, since they do not lead to appreciable differences in the following analysis, will not be discussed. The DOS displays a spin

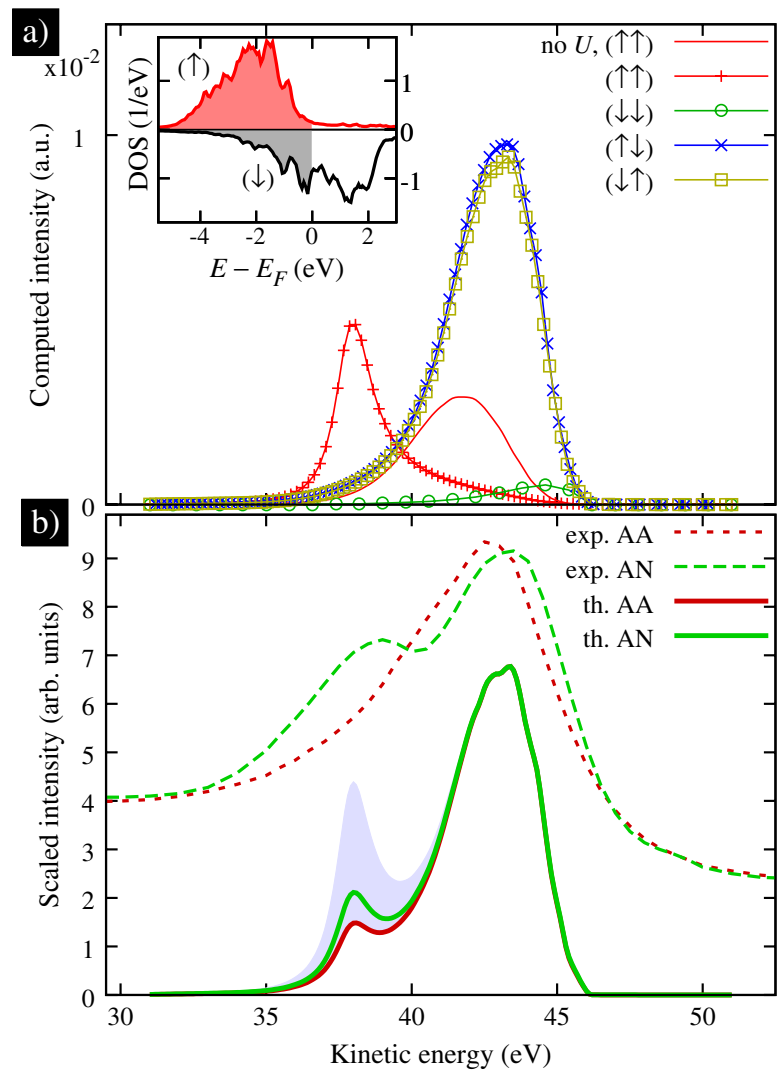

FIG. 3 (color online). (a) Calculated individual APECS contributions in the $A N$ geometry. Lines with symbols sum up to obtain the total spectrum [shown in panel (b)]; the line without symbols indicates the contribution of the $(\uparrow \uparrow)$ component when the hole-hole interaction is neglected. In the inset the computed spin-polarized DOS is shown. (b) Solid lines are the total spectra in the different geometries, normalized to the maximum at about $43 \mathrm{eV}$, compared to experimental data from Fig. 1 (dashed lines). The gray shaded area indicates the variation of the simulated spectrum in the $0-90^{\circ}$ range. 
polarization in the valence band: the $d^{\Uparrow}$ levels fall below the Fermi level $E_{\mathrm{F}}$, resulting in a completely filled majority spin-component band; in contrast, several $(\downarrow)$ spin states fall above $E_{\mathrm{F}}$ resulting in a partially filled minority spin band.

The individual calculated contributions to the Auger transitions for an emission angle of $36^{\circ}$ (as in the $A N$ geometry) are reported in Fig. 3(a). At $E_{k_{A}} \approx 43 \mathrm{eV}$ one finds that the two ( $\uparrow \downarrow)$ and ( $\downarrow \uparrow)$ decays, which include both singlet and triplet states, dominate. A contribution at higher kinetic energy, coming from the ( $\downarrow \downarrow)$ ) channel (triplet), has a low intensity owing to the lower minority spin population and to the partial cancellation between Coulomb integrals in Eq. (3). The curve without symbols derives from the ( $\uparrow$ ) channel (triplet), which falls within the same energy range as the ( $\uparrow \downarrow)$ contributions. It is evident that summing these curves would result in a spectral line shape that gives a good account of the high kinetic energy feature at $43 \mathrm{eV}$, but does not explain the $38 \mathrm{eV}$ peak.

What is missing from this analysis is hole-hole correlation. Since, as seen in the inset of Fig. 3(a), the majority spin band is completely filled and, on the contrary, the minority spin component of the $d$ band is partially filled, $(\uparrow)$ holes are more localized than $(\downarrow)$ ones. As a result, correlation effects can be weak for transitions involving $(\downarrow)$ holes and therefore can be safely neglected while, in contrast, the contribution from the ( $\uparrow)$ term in Eq. (4) (necessarily in a triplet state) should be replaced by an interacting two-hole DOS as proposed by Cini [19] and Sawatzky [20], and recently applied in an ab initio framework for non polarized systems [21]. This is shown, for the case of $U_{\Uparrow}=2.7 \mathrm{eV}$ as pluses in Fig. 3(a), and gives a distinct peak near $38 \mathrm{eV}$.

The sum of the four decay channels is displayed as the solid curves in Fig. 3(b) for the two geometries, and explains all of the features in the data. Following our assignment of the peaks we can describe the low energy $(38 \mathrm{eV})$ peak as a triplet only and the higher energy $(43 \mathrm{eV})$ one as a mixed singlet triplet in agreement to previous works $[11,13,22,23]$. The agreement between measured and computed spectra is very good: Theory well accounts for the presence of two peaks in the Auger energy distribution and their angular dependence in the measurements. The two main features stem from the four spin combinations of the valence electrons involved in the decay: three processes contribute to a single peak and are well described by a single particle approach while, in order to correctly account for the significant shift to lower kinetic energy of the contribution relative to two electrons from the filled majority spin band, a Cini-Sawatzky description has to be used for the second peak. This indicates that hole-hole interaction is more effective when two majority spin electrons are involved and points out how hole-hole correlation strongly depends on the spin properties of the valence electrons.
This investigation demonstrates the coexistence in the same system of two dramatically different on-site electronelectron interactions depending on the spins of the electron couple. These interactions produce clearly separate features in the spin-dependent two-particle density of states probed locally by Auger-photoelectron coincidence spectroscopy and can be unambiguously attributed thanks to angle-resolved measurements. The phenomenon arises because a hole in the completely filled majority spin band is significantly more localized than in the partially filled minority spin one. This different degree of localization should manifest itself in a wide variety of physical properties ranging from one-electron spectral functions, such as those observed in spin-polarized valence band photoemission measurements [24-27], to spin-dependent transport as is relevant for spintronic applications [10].

We thank the staff of the ALOISA beam line at the ELETTRA synchrotron radiation facility for technical support. We gratefully acknowledge financial support from MIUR PRIN 2008 Contract No. 2008AKZSXY, the European Union through the ETSF e-I3 [28] Grant No. INFRA-2007-211956 (G.F.), and the University of Antwerp through the GOA project XANES meets ELNES (F.D.P.). We are deeply indebted to Professor M. Cini and Professor D. Vanderbilt for useful discussions.

[1] W. Kohn, A. D. Becke, and R. G. Parr, J. Phys. Chem. 100, 12974 (1996).

[2] M. Imada, A. Fujimori, and Y. Tokura, Rev. Mod. Phys. 70, 1039 (1998).

[3] G. Kotliar and D. Vollhardt, Phys. Today 57, No. 3, 53 (2004).

[4] L. Hedin and S. Lundqvist, Solid State Phys. 23, 1 (1970).

[5] G. Onida, L. Reining, and A. Rubio, Rev. Mod. Phys. 74, 601 (2002).

[6] H. Jiang, R. I. Gomez-Abal, P. Rinke, and M. Scheffler, Phys. Rev. Lett. 102, 126403 (2009).

[7] T. Das, R. S. Markiewicz, and A. Bansil, Phys. Rev. B 81, 174504 (2010).

[8] H. A. Dürr, G. van der Laan, D. Spanke, F. U. Hillebrecht, and N. B. Brookes, Europhys. Lett. 40, 171 (1997).

[9] F. O. Schumann, C. Winkler, J. Kirschner, F. Giebels, H. Gollisch, and R. Feder, Phys. Rev. Lett. 104, 087602 (2010).

[10] S. H. Mirhosseini, K. K. Saha, A. Ernst, and J. Henk, Phys. Rev. B 78, 012404 (2008).

[11] R. Gotter, F. Da Pieve, A. Ruocco, F. Offi, G. Stefani, and R. A. Bartynski, Phys. Rev. B 72, 235409 (2005).

[12] R. Gotter, A. Ruocco, A. Morgante, D. Cvetko, L. Floreano, F. Tommasini, and G. Stefani, Nucl. Instrum. Methods Phys. Res., Sect. A 467-468, 1468 (2001).

[13] R. Gotter, F. Da Pieve, F. Offi, A. Ruocco, A. Verdini, H. Yao, R. A. Bartynski, and G. Stefani, Phys. Rev. B 79, 075108 (2009).

[14] R. Vollmer and J. Kirschner, Phys. Rev. B 61, 4146 (2000). 
[15] O. Gunnarsson and K. Schönhammer, Phys. Rev. B 22, 3710 (1980).

[16] J.W. Negele and H. Orland, Quantum Many-Particle Systems (Westview Press, Boulder, 1998).

[17] M. I. Trioni, A. Zanetti, G. Fratesi, and G. P. Brivio, Phys. Rev. B 79, 165115 (2009).

[18] S. Achilli, S. Caravati, and M. I. Trioni, Surf. Sci. 601, 4048 (2007).

[19] M. Cini, Solid State Commun. 24, 681 (1977).

[20] G. A. Sawatzky, Phys. Rev. Lett. 39, 504 (1977).

[21] G. Fratesi, M. I. Trioni, G. P. Brivio, S. Ugenti, E. Perfetto, and M. Cini, Phys. Rev. B 78, 205111 (2008).

[22] R. Gotter, F. Offi, A. Ruocco, F. Da Pieve, R. A. Bartynski, M. Cini, and G. Stefani, Europhys. Lett. 94, 37008 (2011).
[23] M. Cini, E. Perfetto, R. Gotter, F. Offi, A. Ruocco, and G. Stefani, Phys. Rev. Lett. 107, 217602 (2011).

[24] A. Santoni and F. J. Himpsel, Phys. Rev. B 43, 1305 (1991).

[25] J. Schafer, D. Schrupp, E. Rotenberg, K. Rossnagel, H. Koh, P. Blaha, and R. Claessen, Phys. Rev. Lett. 92, 097205 (2004).

[26] J. Sanchez-Barriga, J. Fink, V. Boni, I. Di Marco, J. Braun, J. Minar, A. Varykhalov, O. Rader, V. Bellini, F. Manghi, H. Ebert, M. I. Katsnelson, A. I. Lichtenstein, O. Eriksson, W. Eberhardt, and H.A. Dürr, Phys. Rev. Lett. 103, 267203 (2009).

[27] A. L. Walter, J. D. Riley, and O. Rader, New J. Phys. 12, 013007 (2010).

[28] A. Matsuura, N. Thrupp, X. Gonze, Y. Pouillon, G. Bruant, and G. Onida, Comput. Sci. Eng. 14, 22 (2012). 